\title{
Participación ciudadana en Políticas Públicas de Energía: reflexiones para un Chile energéticamente sustentable
}

\author{
Beatriz Hernández P. \\ Universidad Diego Portales, Santiago, Chile. \\ Email: beatriz.hernandez@udp.cl \\ Claudio Huepe Minoletti \\ Universidad Diego Portales, Santiago, Chile. \\ Email: claudio.huepe@mail.udp.cl
}

\begin{abstract}
Resumen: Este estudio analiza el estado actual de la relación entre participación ciudadana y formulación de políticas públicas, y la importancia de la opinión pública a la hora de tomar decisiones políticas relativas al medio ambiente y energía en Chile, usando en particular revisión de encuestas de opinión y entrevistas con expertos. Se analiza si los mecanismos de información y sensibilización ciudadana existentes en este ámbito corresponden a los que permiten una participación más efectiva de la ciudadanía. En ausencia de mecanismos claros de participación, se evalúa las encuestas de opinión pública para ver si alguno de los elementos relevantes de esta opinión, son considerados en las políticas actuales de eficiencia energética. Luego se revisa si los mecanismos de información y sensibilización en temáticas de eficiencia energética han sido apropiados para los objetivos. Se concluye que se requiere importantes innovaciones institucionales y mejores prácticas para poder aspirar a una política pública con más participación relevante de la ciudadanía.

Palabras clave: Participación ciudadana; políticas públicas; opinión pública; eficiencia energética; sensibilización; sociología
\end{abstract}

\section{Citizen Participation in Public Energy Policies: Reflections for an Energy-Sustainable Chile}

\begin{abstract}
This study analyzes the current state of the relationship between citizen participation and public policy formulation, and the importance of public opinion in making policy decisions regarding the environment and energy in Chile, using in particular a review of opinion polls and interviews with experts. The study analyzes whether the existing information and public awareness mechanisms in this area correspond to those that allow for more effective citizen participation. In the absence of clear participation mechanisms, public opinion polls are evaluated to see if any of the relevant elements of this opinion are considered in current energy efficiency policies. Afterwards, we review whether the information and awareness-raising mechanisms on energy efficiency issues have been appropriate for the objectives. We conclude that important institutional innovations and best practices are required in order to aspire to a public policy with more relevant citizen participation.
\end{abstract}

Keywords: Citizen participation; public policy; public opinion; energy efficiency; sensibilization; sociology

\section{A participação cívica nas políticas públicas energéticas. Reflexões para um Chile energeticamente sustentável}

Resumo: O presente estudo realiza uma análise da situação atual da relação entre participação cidadã e formulação de políticas públicas, e a importância da opinião pública na hora de tomar decisões políticas relativas ao meio ambiente e a energia no Chile, mediante recurso a sondagens de opinião e entrevistas com especialistas. Nele é analisado se os 
mecanismos de informação e sensibilização cidadã existentes neste âmbito correspondem àqueles que permitem uma participação mais efetiva dos cidadãos. Na ausência de mecanismos claros de participação, avaliam-se as sondagens de opinião pública para comprovar se algum dos elementos relevantes dessas sondagens são considerados nas atuais políticas de eficiência energética. Em seguida, é examinado se os mecanismos de informação e sensibilização sobre questões de eficiência energética sãos os adequados para os seus objetivos. O estudo conclui que são necessárias importantes inovações institucionais e melhores práticas de modo a ter uma política pública com uma participação mais relevante dos cidadãos.

Palavras-chave:Participação cidadã; políticas públicas; opinião pública; eficiência energética; sensibilização; sociologia.

El consumo energético se ha convertido en un tema de preocupación importante en los últimos años en Chile, tanto por los costos de la energía, como por los problemas de abastecimiento seguro de las necesidades energética y del impacto de la generación y uso de la energía sobre el medio ambiente.

Los estudios sobre política energética en Chile, se han centrado tradicionalmente en el marco normativo y en la institucionalidad, en particular con los cambios derivados de la creación del Ministerio de Energía y de la Agencia chilena de eficiencia energética en el año 2010 (Sierra, 2008).

Si bien hay una variedad de estudios sociológicos sobre los conflictos relacionados con proyectos energéticos específicos (sobre todo eléctricos) que han tenido un gran impacto mediático por sus efectos ambientales y sociales en el territorio donde se ejecutan, los estudios sobre ciudadanía y energía son relativamente recientes, por lo que no existe una literatura demasiado abundante (Bowen et al., 2012; INDH, 2013; Fuenzalida y Quiroz, 2012;Mc Phee, 2010; Tironi 2011; Valenzuela et al. 2012).

En la misma línea de los conflictos energéticos, algunos juristas han estudiado la judicialización de éstos, como resultado de la ausencia de otros mecanismos para resolverlos, ya sean administrativos, por participación ciudadana o por mediación (Couso, 2004; Guiloff, 2011). Por otra parte, Olmedo (2002) argumenta que en Chile, al no haber una norma legal que le dé sentido a la protección del medioambiente consagrada en la constitución, el recurso de protección se constituyó en la principal herramienta de acción judicial en defensa del medioambiente.

En cuanto a la escasa literatura específica sobre participación ciudadana y políticas energéticas, ésta se ha enfocado sobre todo en los procesos de evaluación de impacto ambiental de proyectos de generación eléctrica, los cuales no involucran a toda la sociedad civil sino, generalmente, sólo a los actores afectados directamente y a algunas ONG que actúan como medios de información y sensibilización ciudadana. Estos trabajos, apuntan generalmente a situaciones específicas que afectan a una comunidad particular, así como a sus efectos en la movilización social. En general, los autores realizan una crítica a los procesos de participación, pues se involucra a la ciudadanía en la última fase del proyecto (una vez diseñado) y con un tiempo muy escaso de respuesta (Dufey, 2013; Rodríguez et al., 2013).

No se ha estudiado los mecanismos de información y sensibilización relacionados con la participación ciudadana más allá de proyectos específicos, que podría abrir el debate al conjunto de necesidades de los ciudadanos, considerando la diversidad territorial, socioeconómica, demográfica, etaria o de intereses y necesidades. ${ }^{1}$ Tampoco se ha estudiado si efectivamente y por qué medio la opinión ciudadana se transforma efectivamente en un aporte a las políticas públicas.

Desde el surgimiento de temas ambientales en los años 60 la participación ciudadana ha estado necesariamente ligada al “descontento verde” que se tradujo en múltiples protestas por decisiones que les afectaban directamente, como por ejemplo el uso del suelo o el desarrollo de energías contaminantes (Lauwers, 1983). Ante los crecientes conflictos locales y la oposición de grupos medioambientalistas algunos gobiernos acordaron nuevas formas de consulta y participación ciudadana en los años 80 bajo el principio de “participación pública efectiva”2 que finalmente se plasmaron en el acuerdo de la Cumbre de Río de 1992 (Principio 10) "El mejor modo de tratar las cuestiones ambientales es con la participación de todos los ciudadanos interesados, en 
el nivel que corresponda. Los Estados deberán facilitar y fomentar la sensibilización y la participación de la población poniendo la información a disposición de todos. ${ }^{3}$ " Así como en la Convención Aarhus de 1998 sobre el acceso a la información, participación pública en procesos de toma de decisiones y el acceso a la justicia en decisiones medioambientales, actualmente firmada por 56 países $^{4}$.Podemos inferir a partir de estos acuerdosque la falta de participación ciudadana en el proceso de formulación e implementación de políticas públicas desencadena mayores conflictos en las instituciones políticas.

Pese a la creciente presencia en la discusión pública del tema energético y medio ambiental en Chile, los ciudadanos no han tenido una participación particularmente activa en las políticas: ni en su diseño ni en su implementación. El proceso de planificación participativa para la política energética de largo plazo - Energía $2050^{5}$, fue considerado el primero en incluir participación ciudadana en el año 2015 con una consulta pública. Sin esta participación, las políticas dependen fundamentalmente de expertos, y, además, muchas acciones que dependen de decisiones individuales se vuelven difíciles de implementar.

De lo anterior, surgen varias preguntas respecto al vínculo entre el ciudadano y las políticas energéticas. Por ejemplo: ¿Es posible convertir a los chilenos en partícipes activos respecto a la estrategia de desarrollo energético nacional y por qué mecanismos? ¿Qué mecanismos de información y sensibilización pueden involucrar mejor al ciudadano en el proceso de formulación de políticas públicas en torno a temas energéticos? ¿Cómo puede transformarse la opinión pública en un elemento relevante para las políticas públicas?

El presente estudio busca analizar los vínculos entre mecanismos de información y sensibilización, la participación ciudadana y la opinión pública en el proceso de toma de decisiones delas políticas públicas de energía, en particular de eficiencia energética. Se trata de reflexionar sobre el vínculo entre la información ciudadana y las políticas públicas en energía (específicamente en eficiencia energética), apuntando en particular a identificar elementos que puedan mejorar las políticas adecuándolas a la diversidad de contextos, requerimientos y objetivos.

Como ejemplo específico del vínculo mencionado, se analizan políticas de eficiencia energética dada su relación con las diversas dimensiones de la política (ambiental, económica y social). La eficiencia energética es vital para reducir el costo económico del consumo de energía y para lograr los objetivos de reducción de emisiones de gases de efecto invernadero. La agencia internacional de energía estima que del orden del $40 \%$ de la reducción de emisiones requerida para alcanzar al 2050 el límite global de alza de temperatura de 2 grados Celsius, vendría potencialmente de la eficiencia energética (AIE, 2015a). Sin embargo, lograr este potencial implica la participación de millones de actores (individuos y organizaciones), de modo que su involucramiento activo es fundamental para que las políticas funcionen adecuadamente.

La eficiencia energética se refiere al uso técnicamente eficiente de la energía, es decir a la optimización del consumo energético para producir bienes y servicios. Según la Agencia Internacional de Energía (2015b), algo "es más eficiente energéticamente si entrega más servicios consumiendo la misma cantidad de energía, o los mismos servicios consumiendo menos energía" .

La eficiencia energética impacta sobre los costos reales de empresas y sobre los gastos de los hogares, permite además mejorar procesos y gestión en general además de aportar a la innovación. La eficiencia energética tiene impactos ambientales positivos al reducir el consumo de combustibles, y de ese modo las emisiones, y materiales y además exige generar información relevante y tiene un impacto en la formación ciudadana pues releva la importancia de la energía en la acción cotidiana. Pero también tiene una alta complejidad "técnica” y de implementación asociada (requiere cambios de conducta y estructurales en muchos actores), lo cual lo hace un desafío relevante y complejo a la vez.

La metodología que se emplea en esta investigación es cualitativa. Para estudiar los vínculos de la participación ciudadana con las políticas energéticas y con la información y sensibilización, se contrastan los resultados de diversas encuestas de opinión pública y se realizan entrevistas a actores clave de las instituciones públicas respecto a la información y sensibilización en materia de energía. Se estudian las estrategias de infor- 
mación y sensibilización ciudadana, los sistemas de información institucionales para analizar sus efectos en la participación ciudadana, así como el aumento del activismo y la preocupación por temas medioambientales y energéticos.

Goodin, Moran y Rein (2006), señalan que la opinión pública es uno de los elementos centrales en la formulación de políticas. Diversos autores han estudiado la importancia de las encuestas públicas como medios de expresión y también como estímulo para la participación ciudadana en el debate público (Price, 1994). La opinión pública se considera, por lo tanto, un eje de articulación entre la información y sensibilización y las políticas.

En Chile, son pocas las encuestas de opinión pública que incluyen preguntas sobre energía (sustentable) y tampoco existe una continuidad temporal. En consecuencia, este estudio usa a menudo el aspecto ambiental de la opinión y prácticas ciudadanas, porque es un aspecto central que se enfatiza para promover la eficiencia y sobre el cual hay más estudios de opinión.

Para llevar adelante este trabajo, en primer lugar, se revisan conceptos y enfoques sobre la participación ciudadana y se ven los posibles vínculos entre información, participación, opinión pública, y políticas. La segunda parte explora las características de la opinión del ciudadano en temas de energía y medio ambiente, apuntando en particular a la coherencia o inconsistencias en la opinión expresada. Dado que no hay mucha información específica sobre opinión pública respecto a eficiencia energética, esta revisión abarca un ámbito un poco más general.

Posteriormente, se examinan las estrategias que se han aplicado en materia de información ciudadana su evolución, alcances y limitaciones a partir fundamentalmente de entrevistas realizadas a actores sectoriales.

Por último, se concluye reflexionando sobre los mecanismos existentes de información y su potencial para alcanzar el objetivo de una participación efectiva en el diseño e implementación de políticas públicas.

\section{Las escaleras de participación en las políticas públicas}

Para la OCDE hay que fortalecer las relaciones entre las instituciones públicas y los ciudadanos a través de la participación activa en tres dimensiones: información, consulta y coparticipación. Esto porque ofrecen una mejor base para elaborar políticas públicas de calidad y con procesos de toma de decisiones más eficaces, transparentes y confiables (OCDE, 2006). La participación en diversos países de la OCDE se da desde una etapa inicial del proceso de diseño de las políticas públicas tanto a nivel de información como de consulta, lo que permite un diálogo en el que se pueden formular preguntas y dar respuestas de manera bidireccional.

En general, los estudios sobre la participación ciudadana en temas energéticos han identificado a la información y sensibilización ciudadana como un primer paso en de la participación (Montero, 2011). Para ello, se han hecho estudios de caso caracterizando, en primer lugar, a los actores sociales y sus necesidades de información (Rau, 2013).Cabe notar que en la información hay dos tipos de actores involucrados: por un lado, los responsables de difundir la información (instituciones públicas de medio ambiente y energía, ONGs, redes sociales) y, por otra, los ciudadanos como receptores.

Según Rau et al. (2012), existen etapas claras para conseguir un mayor grado de involucramiento en los procesos, buscando otorgar mayor transparencia a las políticas públicas y sentido de justicia de éstas en los ciudadanos. Para diversos autores, la información es el primer paso en la llamada escalera de participación ciudadana (Arnstein, 1969) que asciende desde menos a más involucramiento. Lo relevante, entonces, para que la información sea incidente sobre las políticas es dar espacio de participación real a los ciudadanos los que requieren como base mecanismos adecuados de información y consulta. 
Figura 1.

\section{La Escalera de Participación Ciudadana y sus Dos Tipos de Actores}

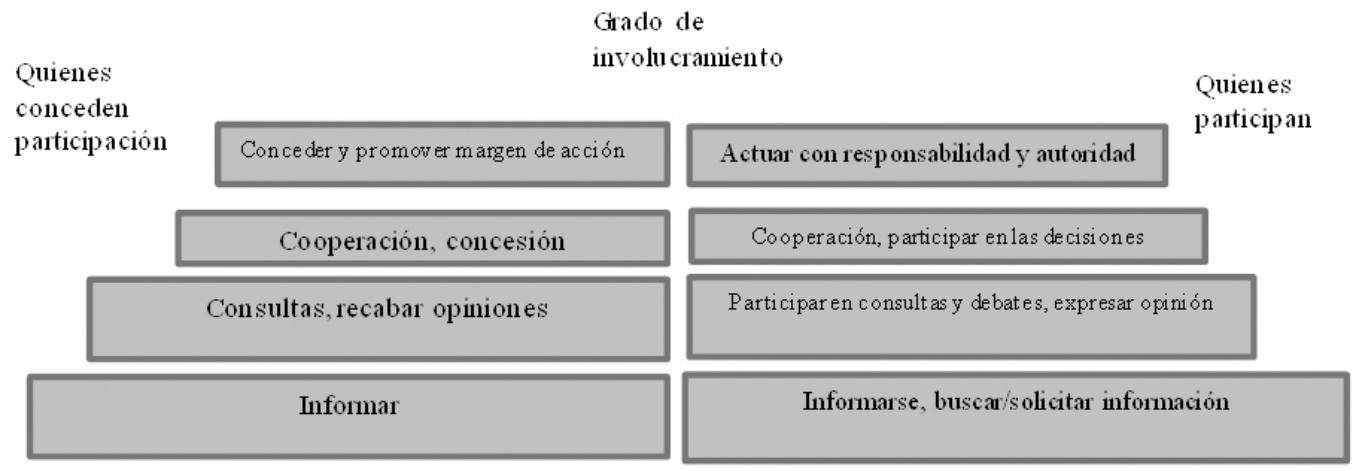

Fuente: Elaboración propia basada en Rau et al. (2012)

En la actualidad hay un mayor consenso entre los gobiernos de países desarrollados de optar por mecanismos de participación no vinculantes pero activos, según sugiere la OCDE. Hay diversas visiones sobre la “escalera” de participación ciudadana (ver figuras 1 y 2), pero en todas ellas la OCDE entiende por participación activa aquella que genera co-participación, es decir en que los ciudadanos "participan en el establecimiento de la agenda, y contribuyen a dar forma al diálogo con la administración pública” (OCDE, 2006, p. 67).

Algunos autores critican los mecanismos de consulta que se reducen a una participación "simbólica" (Arnstein, 1969) o de "fachada" (Prieto Martín, 2010). Sus propuestas buscan empoderar al ciudadano con una opinión vinculante en el proceso de toma de decisiones. Sin embargo, ni siquiera los países más avanzados en participación ciudadana (los países nórdicos, por ejemplo) han llegado a ese poder de decisión.

Figura 2.

Escaleras de Participación Ciudadana

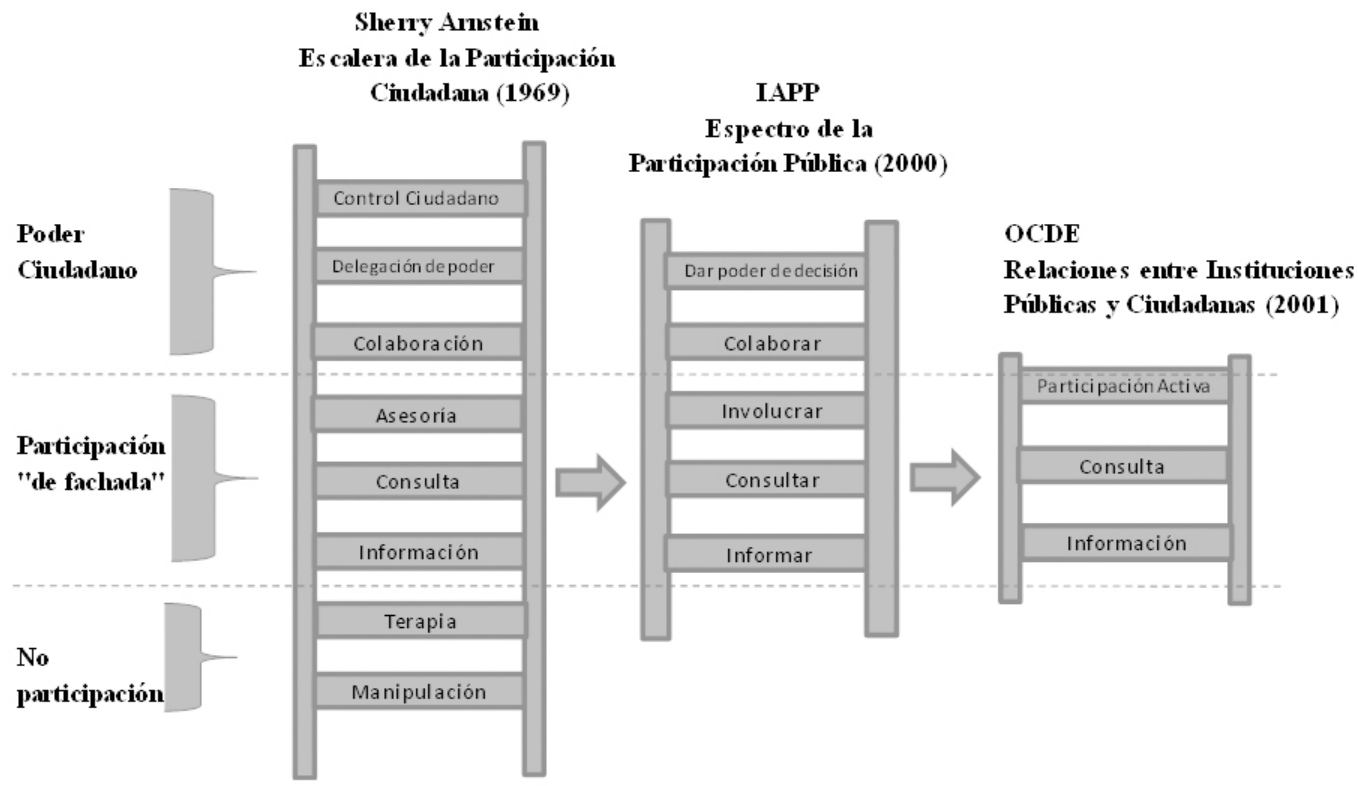

Fuente: Elaboración propia con datos de Prieto-Martin (2010). 
Mayor información puede ayudar, pero no redunda automáticamente en una mayor participación ciudadana (activa) en los procesos de políticas, que equivaldría a "subir" a los peldaños superiores de la escalera. Además, muchos gobiernos tienden a generar flujos de información unidireccionales -desde "arriba" hacia "abajo"- sin mecanismos de retroalimentación ni espacio de negociación, por lo que los ciudadanos no tienen realmente ninguna capacidad de influir en el proceso de toma de decisiones, y se quedan en los niveles de catarsis, muy lejos de las formas de participación más exigentes, que corresponden a la co-gestión.

La influencia de la información sobrecambios en la participación ciudadana ha sido destacada por varios autores como Castells, quien señala que "la comunicación es el espacio donde se construyen las relaciones de poder, el poder para actuar” (Castells, 2010, p.121). En el caso de Chile además se destaca que, a mayor uso de las redes sociales como Facebook y Twitter, "los usuarios tienen 11 veces más probabilidades de participaren marchas en la calle” (Valenzuela et al, 2012, p. 20).El acceso a la información desde las instituciones internacionales, nacionales, locales, de la sociedad civil y de educación se ha convertido en una condición clave de la construcción de las identidades colectivas (Martín-Barbero, 2000).

Como señala Montero, las inquietudes de los ciudadanos no son valores estáticos, sino que registran acusados movimientos de un año a otro, en función, sobre todo, de aquellas parcelas que han marcado la agenda política o se han reflejado con insistencia en los medios de comunicación (Montero, 2011). Castells indica, por su parte, que

Lo que está realmente cambiando es que el espacio público está siendo transformado en todas las sociedades. El espacio público es el espacio donde la sociedad delibera, construye sus percepciones y decisiones políticas y entonces, a partir de ahí se crea el sistema de decisión social y político. (Castells, 2010, p.144).

Para Montero (2011), la diferencia entre receptores pasivos y activos viene determinada por las situaciones de discriminación tecnológica que limitan el acceso de las clases menos favorecidas a estas nuevas herramientas de comunicación, aunque habría que añadir que la brecha viene también determinada por los niveles de educación, puesto que la información debe ser entendida y procesada para generar conocimiento. Adicionalmente, es importante determinar el tipo de información pertinente para cada actor. En el sector energético, la complejidad de los aspectos técnicos relevantes, dificulta la comprensión de varios temas para el ciudadano común, contribuyendo a la falta de información relevante para participar.

Mc Phee (2010), citando a Padilla y San Martin (1994) y Claude (1997), señala que

“(...) el surgimiento de los conflictos ambientales, o de otra índole, pero que utilizan la retórica ambientalista, se enmarca dentro del contexto de implementación de la economía neoliberal en los distintos países de Latinoamérica, que en el caso chileno ocurre en un escenario de dictadura y post-dictadura (...) de este modelo económico se ha presionado de manera importante los recursos naturales y con ello desencadenado importantes procesos de contaminación y/o degradación ambiental, ante los cuales han surgido respuestas y movilizaciones ciudadanas con el propósito de defender el medioambiente” (Mc Phee, 2010, p. 43).

La ausencia de participación ciudadana (activa) en la etapa de desarrollo de proyectos ha favorecido la protesta (reactiva) y los conflictos. Los países que han buscado avanzar en participación ciudadana, han desarrollado metodologías que permiten involucrar a los ciudadanos en el establecimiento de las prioridades de la acción pública a nivel nacional de una manera mucho más activa. Desde el año 2003 tanto la Unión Europea, Canadá y Estados Unidos incluyeron o modificaron legislaciones para incorporar procesos de participación ciudadana $^{7}$ como las consultas tal y como se establecía en la Convención Aarhus. Algunos países han llegado más lejos y están implementando una coparticipación con estrategias de grupos de trabajo abiertos, paneles de no-especialistas y procesos de diálogo. Por ejemplo en Dinamarca y Noruega se forman Grupos de Discusión en los que 10 o 15 ciudadanos (elegidos de manera aleatoria) interrogan a los expertos sobre las políticas a adoptar y presentan al final públicamente sus conclusiones consensuadas; y los Jurados Ciudadanos en Francia que es un interrogatorio a expertos en forma de Tribunal abierto al público pero sin necesidad de consensuar las 
conclusiones (OCDE, 2006). De acuerdo a Abaza et al. (2004) esto permite un análisis conjunto y un control sobre el proceso de toma de decisiones y su implementación.

El Reino Unido, por ejemplo, desarrolló un plan en el marco de la reforma al Servicio Civil ${ }^{8}$ para incrementar la participación en la generación de políticas públicas por medio de dos mecanismos: "elaboración abierta de políticas" (open policy-making) y "elaboración disputable de políticas” (contestable policy making) que se consideran claramente distintas al modelo de consulta pública.

En Chile desde hace algunos años existen algunos mecanismos para participar en políticas públicas ${ }^{9}$, en particular en los ámbitos de planes y normas, que son sometidos a consulta además de la participación para proyectos específicos que existe desde la Ley de Bases del Medio Ambiente (1992), en el contexto de los estudios de impacto ambiental. Aunque son mecanismos bastante acotados de consulta (cabrían dentro de la "participación de fachada" en la "escalera de participación” porque se dan una vez propuesta la política), según la encuesta del Ministerio del Medio Ambiente, el 58\% de los encuestados considera que en Chile existen espacios para participar en políticas públicas de medio ambiente (Ministerio de Medio Ambiente, 2014).

Hasta el gobierno de Michelle Bachelet el Ministerio de Energía no tenía una oficina de información con el argumento de que por su carácter técnico este Ministerio no tenía una relación con la ciudadanía de manera directa y se encontraban con dos barreras (1) la complejidad del lenguaje y de la materia a tratar y (2) la escasa cultura de participación que hay en Chile (Zulueta, 2014). Esto

"porque los ciudadanos solo se interesaban por el precio de la luz y lo demás era un terreno árido y desconocido, en las mesas de trabajo solo participan expertos, y sabemos que en Chile especialmente en temas de energía la masa crítica es mínima, son muy pocos, con pocas capacidades y considerando el terreno que tiene el Ministerio actualmente los actores son siempre los mismos" (Duffey, 2014).

Por otra parte, aunque cada vez se accede más a la información a través de redes sociales y hay mayor conectividad (internet y telefonía móvil), no se ha alterado el carácter vertical en que se ha construido la relación Estado-individuo (Informe de Desarrollo Humano PNUD, 2008).

Además, parece que los ciudadanos que buscan información oficial sobre temas de relevancia ambiental (como la eficiencia energética) suelen tener un perfil socioeconómico similar. De acuerdo a los datos aportados por la Oficina ministerial de atención a la ciudadanía de la Subsecretaría de Energía, aunque no existe un registro sistematizado de las consultas se ha observado que la mayoría de las consultas sobre información medioambiental son hechas desde el mundo empresarial y universitario.

Lo anterior sugiere una brecha socioeconómica en el uso de las nuevas tecnologías y, por tanto, en el acceso de la información y también una brecha generacional, que obliga a pensar en modalidades de acercamiento a todos los sectores de la población. Esta brecha agrega una capa adicional a la desigualdad: esta no sólo se manifiesta en la exclusión en el acceso alos servicios públicos y privados, sino también en una asimetría en la información que las personas reciben sobre los mismos. Como señala Montero (2011), el conocimiento debe aprenderse siempre por la acción; es decir los ciudadanos requieren información rigurosa y asequible con la que asumir su responsabilidad y poder contribuir al esfuerzo colectivo.

Otro aspecto que apuntaría a la mayor participación de la juventud, serían los valores "post materialistas". Con datos comparados a nivel mundial, el sociólogo Ronald Inglehart (1990) diseñó un conjunto de conceptos e indicadores para analizar los valores y sus transformaciones en sociedades democráticas industrializadas, fundamentalmente para observar los cambios desde una tendencia cultural "materialista" a una "post materialista", es decir, desde valores centrados en la seguridad física y económica, hacia otros valores que se centran en la libertad y autonomía, la autoexpresión y la calidad de vida. La aparición de estos valores sucede cuando las necesidades materiales de la población están ya aseguradas, por lo que se avanza hacia nuevas preocupaciones inmateriales. 
Para este autor la propagación de los valores postmaterialistas está empíricamente vinculada a la democratización política ${ }^{10}$. Desde la perspectiva de la elección racional (Downs, 1957), para poder comprometerse activamente con la política se necesitan recursos, tales como tiempo, dinero y habilidades cognitivas. Por lo tanto, reducir estos costos aumentará la probabilidad de participar en política. Esto también podría reflejar la mayor participación actual de la juventud.

Para Valenzuela "la participación motivada por el deber cívico ha sido reemplazada por la participación motivada por metas de autorrealización personal y colectiva” (Valenzuela et al,2012, p.20).

Además,

“[para los jóvenes] el espacio de referencia no [es] la nación ni el Estado-nación, sino el vínculo más directo entre espacios locales y movilizaciones globales. No aspiran a ver allí cumplidas reivindicaciones materiales (empleo, ingresos) o de poder (cuotas en partidos, representación parlamentaria), sino que se movilizan por causas más genéricas y universalmente compartibles, como la paz mundial, los derechos humanos, la justicia, la defensa del medio ambiente, y otras” (CEPAL y OIJ, 2007, p. 273).

En síntesis, hay un vínculo general entre información y participación y políticas, si bien ciertas características (rango etario, situación socioeconómica) son determinantes del carácter del vínculo.

A continuación se revisan los antecedentes sobre opinión pública chilena en materia de eficiencia energética (energía y medio ambiente) y sobre los mecanismos de información y sensibilización existentes actualmente.

\section{Opinión Pública en Energía y Medioambiente en Chile}

Cada vez con mayor frecuencia, se han aplicado encuestas de opinión en Chile para conocer los temas más importantes que deben estar en la agenda pública. El tema energético no ha sido una presencia regular en las principales encuestas, aunque si se considera su relación con los temas ambientales (en particular para la eficiencia energética), hay un poco más de información relevante sobre opinión pública, aunque aún escasa. Por ello, se revisa también la opinión en temas ambientales.

Como se ha señalado, estas encuestas son importantes porque no sólo reflejan la opinión ciudadana, sino que también abren la discusión pública, permitiendo conocer las percepciones de los miembros de una comunidad diversa (si bien no empoderan al ciudadano, pues sólo se les consulta). Además, resulta relevante también conocer el perfil de las personas que se interesan más en temas de energía y medio ambiente y cuáles son sus motivaciones.

Como indica Price (1994), la opinión pública se trata de juicios colectivos que no se realizan dentro de la esfera del Estado, pero que afectan las decisiones políticas; por tanto, su papel es clave en la formación de un público más activo y participativo en los asuntos públicos.

La Encuesta Nacional UDP del año 2007, contiene temas medioambientales, pero no volvió a incluir preguntas de este tipo hasta el año 2014. Las encuestas de IPSOS en abril del 2011,contiene temas sobre energía nuclear e hidroeléctricas; en noviembre 2011 contiene una pregunta sobre Hidro Aysén y, en abril del 2012,pregunta sobre consumo y gasto agregado por mantener el medioambiente. Por su parte la Encuesta CEP en sus series 2000 y 2010, incluye preguntas sobre la protección al medioambiente,y en su versión septiembre - octubre 2013, además de tener la opción “medioambiente”, incluyó la opción “energía” en la pregunta:¿Cuáles son las tres problemas a los que deberían dedicar el mayor esfuerzo en solucionar el Gobierno?"11

La Primera Encuesta Nacional de Medio Ambiente (2014), muestra que los estratos socioeconómicos más altos (C1) son más críticos con la poca exigencia en las regulaciones medioambientales y la educación sobre el medio ambiente que se da en el país. Al abordar con los entrevistados su grado de acuerdo o desacuer- 
do, es relevante mencionar que un $85 \%$ de los entrevistados está de acuerdo o muy de acuerdo con que proteger el medio ambiente genera ahorros, pero al mismo tiempo un $51 \%$ cree que proteger el medio ambiente encarece el costo de vida, pero un aspecto positivo es que se tiene la percepción de que en las escuelas los niños(as) y jóvenes aprenden a cuidar elmedio ambiente (60\%). Por otra parte un $66 \%$ de las personas está muy en desacuerdo o en desacuerdo de que en el país se le da importancia suficiente a la educación ambiental y un $60 \%$ no está de acuerdo en que Chile tiene regulaciones exigentes en temas medio ambientales, según detalla el cuadro 1.

Cuadro 1.

Percepción de cuidado medio ambiental por sexo, edad, tramo socioeconómico y zona del país ¿Cuán de acuerdo está usted con las siguientes frases...?

Respuesta Muy de Acuerdo-De acuerdo (expresado en \%)

\begin{tabular}{|c|c|c|c|c|c|c|c|c|c|c|c|c|}
\hline & \multirow[b]{2}{*}{ Total } & \multicolumn{2}{|c|}{ Sexo } & \multicolumn{3}{|c|}{ Edad } & \multicolumn{4}{|c|}{$\mathrm{GSE}$} & \multicolumn{2}{|c|}{ Zona } \\
\hline & & $\begin{array}{l}\text { Hombre } \\
\mathrm{N}: 2: 029\end{array}$ & $\begin{array}{l}\text { Mujer } \\
\text { N.3028 }\end{array}$ & $\begin{array}{l}18-34 \\
N: 1464\end{array}$ & $35-54$ & $55 y+$ & $\begin{array}{c}\mathrm{C}_{1} \\
\mathrm{~N}: 7 \mathrm{~g}_{5}\end{array}$ & \begin{tabular}{c|}
$\mathrm{C}_{2}$ \\
$\mathrm{~N}$
\end{tabular} & $\mathrm{c}_{3}$ & $\begin{array}{c}\mathrm{D}-\mathrm{E} \\
\mathrm{N}:-447\end{array}$ & $\begin{array}{c}\text { Santiag } \\
\mathrm{N} 542\end{array}$ & $\begin{array}{l}\text { Regiones } \\
\text { N:44:4:5 }\end{array}$ \\
\hline $\begin{array}{c}\text { Froteger el } \\
\text { medio } \\
\text { ambiente } \\
\text { genera ahomos }\end{array}$ & 85 & 85 & 85 & 86 & 84 & 83 & 88 & 85 & 87 & 82 & 85 & 85 \\
\hline $\begin{array}{c}\text { En las escuelas } \\
\text { los niños y } \\
\text { jovenes } \\
\text { aprenden a } \\
\text { cuidar el medo } \\
\text { ambiente }\end{array}$ & 60 & 60 & 62 & 61 & 61 & 61 & 54 & 55 & 62 & 64 & $5^{6}$ & 64 \\
\hline $\begin{array}{c}\text { Froteger el } \\
\text { medio } \\
\text { ambiente } \\
\text { encarece el } \\
\text { costo de vida }\end{array}$ & 51 & $5^{2}$ & 51 & 47 & 52 & 55 & 43 & $4^{2}$ & $4^{8}$ & $5^{8}$ & $5^{2}$ & 50 \\
\hline $\begin{array}{l}\text { Chile tiene } \\
\text { regulaciones } \\
\text { exigentes en } \\
\text { temas } \\
\text { ambientales }\end{array}$ & 34 & $3^{6}$ & $3^{2}$ & 33 & 30 & 41 & 24 & 27 & 33 & 40 & $3^{2}$ & 35 \\
\hline \begin{tabular}{|c|} 
Se da la \\
importancia \\
suficiente a la \\
educación \\
ambiental en \\
nuestro pás
\end{tabular} & $3^{x}$ & $3^{1}$ & $3^{1}$ & 27 & 29 & 40 & 16 & 21 & 28 & 41 & 28 & 34 \\
\hline
\end{tabular}

Fuente: Elaboración propia en base a datos del Ministerio de Medio Ambiente (2014) ${ }^{12}$

Desde el año 2010 se aprecia que los ciudadanos manifiestan una mayor preocupación tanto por la protección del medio ambiente como por la inseguridad del abastecimiento y los precios de los recursos energéticos (gas y petróleo) en diversas encuestas. La encuesta CEP de ese año, indica que un $45 \%$ demandaba una mayor información y educación a la ciudadanía sobre estos temas y un 32\% creía que "fuertes multas a las personas que dañen el medio ambiente" son la mejor forma para lograr que las personas y sus familias en Chile protejan el medio ambiente. 
De acuerdo a la Encuesta de Tren Digital, en el 2009 solo 21,9\% asistió a algún evento o manifestación pública sobre el tema e, incluso, sólo el 34,4\% se unió a un grupo de protesta en Facebook. Asimismo, en la encuesta del CEP 2010 un 30\% estaba dispuesto a hacer sacrificios significativos para proteger el medio ambiente (por ejemplo, pagar más impuestos, mayores precios o bajar su nivel de vida).En los últimos años nos encontramos con mayores expresiones de protesta pública en temas ambientales, que se involucran en redes de acción comunitaria y local usando contactos personales y tecnologías interactivas (Valenzuela, 2012; RaynesGoldiey Walker, 2008).

El 71\% de los jóvenes declaran informarse periódicamente con las redes sociales y medios de comunicación en línea (periódicos digitales) ya que pueden consultarlo varias veces al día desde cualquier hora y lugar (Tren Digital, 2013). Por lo que el uso de internet como fuente de información podría predecir la participación de ciudadanos en manifestaciones.

Un estudio de Aldunate et al. (2011, p.26) recalca que los determinantes estadísticamente significativos para participar en manifestaciones son: la pertenencia a grupos cívicos, el interés en la política, los valores post materiales y el uso de Facebook y de Youtube. Sin embargo, aunque la sensibilización se limita sobre todo a unirse a grupos de protesta en Facebook y ver videos en Youtube.

De acuerdo a Illanes el 28,3\% de los chilenos ha participado en una marcha y un 30,2\% ha participado en foros sobre temas educacionales y energéticos en el 2012 (Illanes, 2013, p.5). Estos datos son superiores a los que presenta la Encuesta Social General de Canadá en el año 2013 para ciudadanos de más de 25 años, en el que poco más del 15\% participaba en redes sociales y menos del 10\% participaba en una marcha. Este último país garantiza la participación de los ciudadanos en el proceso de toma de decisiones en temas medioambientales desde el año 2003 para resolver institucionalmente los conflictos en este ámbito.

El caso de Hidroaysén refleja la importancia de tener mayor transparencia y acceso a la información sobre la opinión pública. En el año 2008 un 54,7\% de los ciudadanos estaba a favor de la construcción de la central hidroeléctrica en Aysén mientras que el 2011 la aprobación sólo llegaba al 29,1\%; es decir, un 61,1 \% desaprobaba el proyecto (el detalle de la evolución de la opinión pública se encuentra en el gráfico 1).Este cambio en la opinión pública coincide con un período de intensa difusión de información sobre el proyecto por grupos detractores de la iniciativa.

\section{Gráfico 1.}

Posición a favor o en contra a la construcción de centrales hidroeléctricas en la Región de Aysén ${ }^{13}$

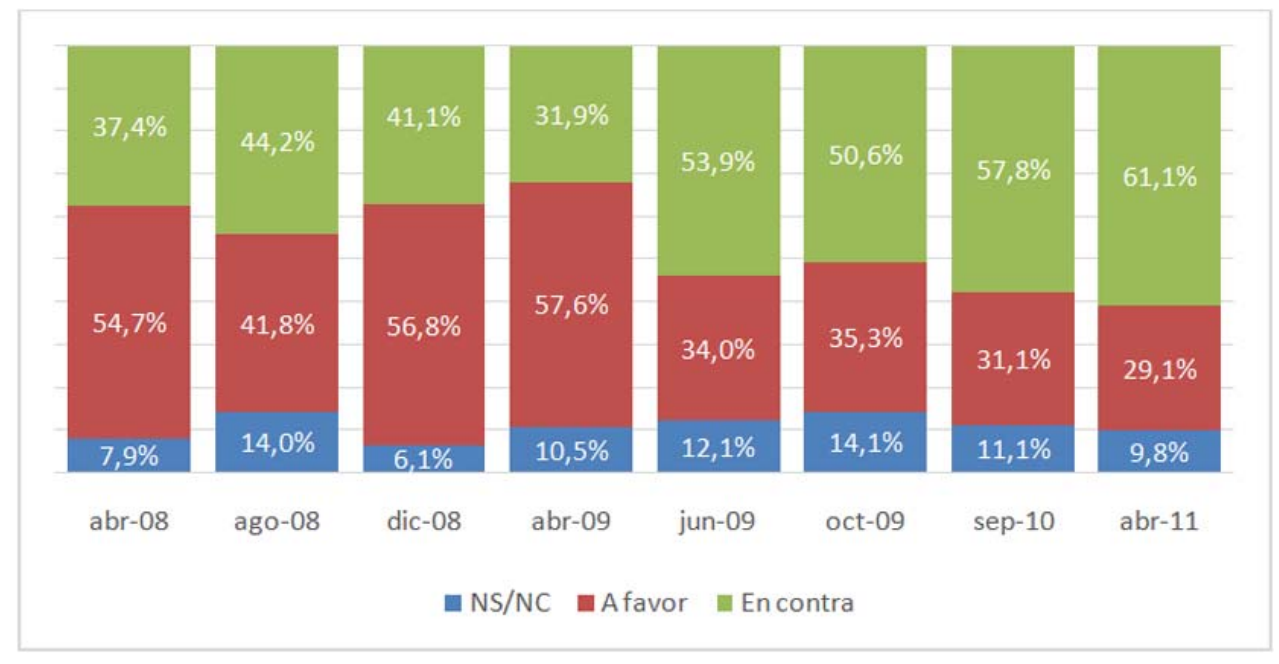

Fuente: Elaboración propia con datos de IPSOS (2011) 
Como se puede observar, los cambios en el activismo en los últimos años se han ido manifestando en las encuestas de opinión pública, teniendo un punto de inflexión clave con el caso emblemático de Hidro Aysén desde el 2010. Las ONGs y ciudadanos se movilizaron en las redes sociales para fomentar la información y sensibilización creando un fenómeno social de protesta. Los medios de comunicación tradicionales (radio y televisión) también dieron una gran cobertura mediática a las demandas ciudadanas, lo que fomentó una participación creciente ${ }^{14}$.

De acuerdo a las encuestas de la UNAB 2013 y 2014 los más preocupados por el medio ambiente son las clases medias o nivel socioeconómico ${ }^{15} \mathrm{C} 3$, ya que fueron los más críticos con la gestión del gobierno en casos puntuales de impacto medioambiental como los proyectos en Hidroaysén, Castilla, Pascua Lama y Ventanas; siendo los menos críticos el nivel ABC1. Sin embargo, el nivel socioeconómico C3 no parece particularmente dispuesto a pagar más por productos que cuidan el medio ambiente como se aprecia de la encuesta IPSOS $2013^{16}$ se ve que esto no ha cambiado mucho en los últimos años, ya que la encuesta IPSOS 2013, tampoco muestra de parte de los encuestados un compromiso para asumir grandes costos individuales en la protección del medio ambiente(ver gráfico 2).

\section{Disposición a Pagar por un Producto que Cuida el Medio Ambiente}

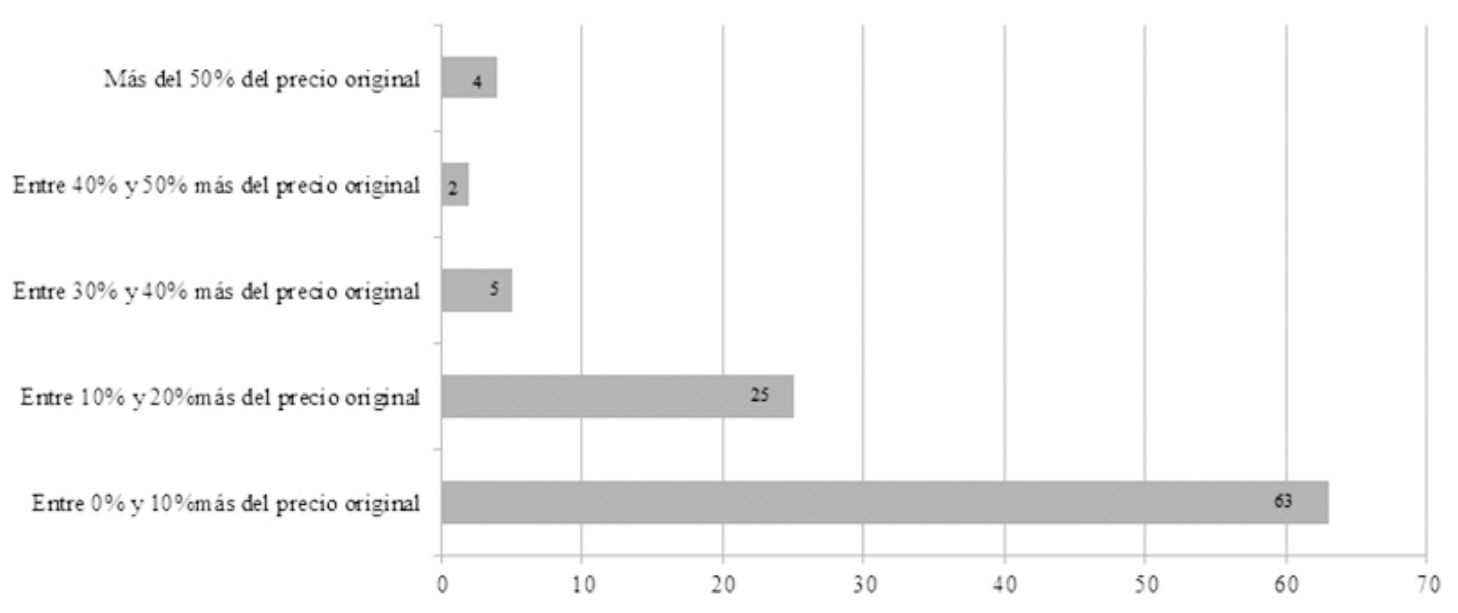

Fuente: Elaboración propia con datos de IPSOS $(2013)^{17}$

En la encuesta UDP 2014,se observa un avance en la conciencia medioambiental, ya que un 18,6\% señala haber apoyado activamente o con dinero causas ambientales y un 53,8\% declara que, aunque no lo ha hecho, lo haría en un futuro. Es más, los encuestados también dan prioridad al medio ambiente sobre el crecimiento económico y estarían dispuestos a prohibir proyectos energéticos. Esto es consecuente con el rechazo quehan generado ciertos proyectos energéticos y a la falta de protección del gobierno hacia el medio ambiente, cuestionando así el modelo económico imperante (ver gráfico 3). 


\section{Cuestionamientos al crecimiento económico sin consideración ambiental}

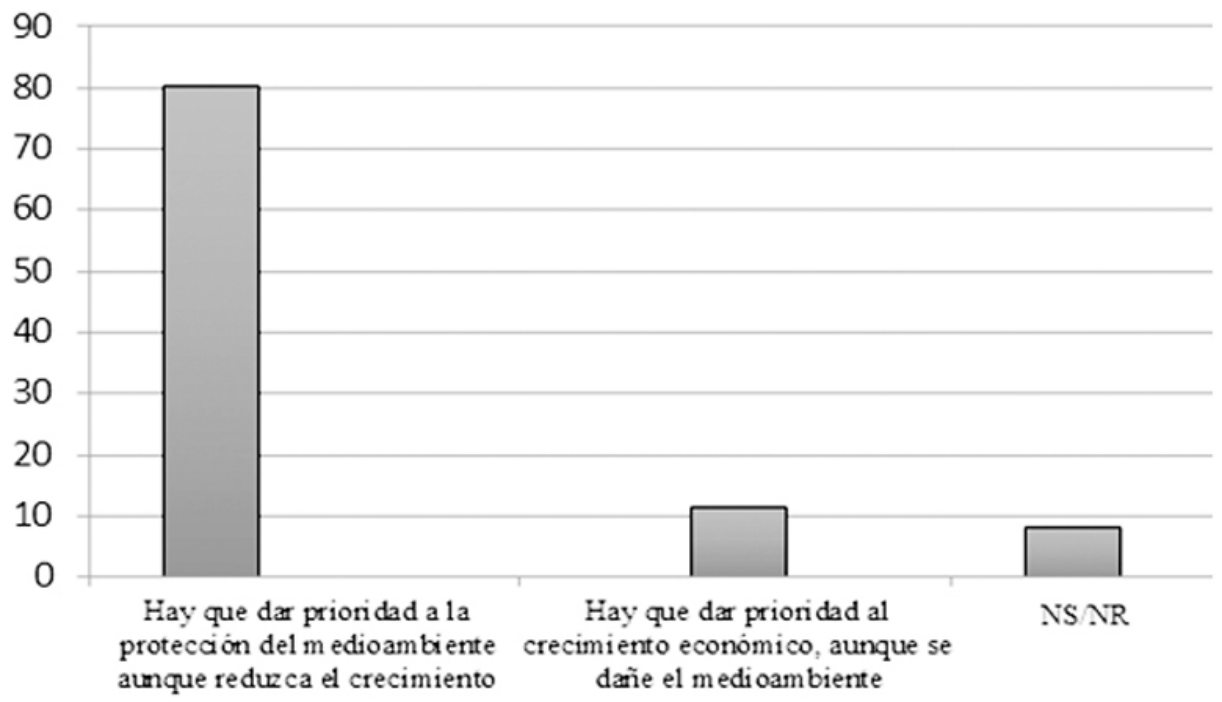

Fuente: Elaboración propia con datos de Encuesta Nacional UDP $2014^{18}$

Sin embargo, aunque los ciudadanos, en particular los jóvenes, se informan cada vez más y algunos participan en manifestaciones ciudadanas ${ }^{19}$, estas acciones colectivas no se reflejan necesariamente en acciones individuales para cambiar hábitos de vida.

De acuerdo a la encuesta GFK/Adimark, Reciclaje en Chile (2015), el número de chilenos que recicla alcanza solo el $17 \%$ como se detalla en el gráfico 4. Sólo una pequeña parte de la población recicla (y hay además una disminución respecto al $29 \%$ total en el 2012). Las razones para no reciclar son variadas. Ciertamente, los segmentos que más reciclan (ABC1 23\% y C2 20\%) son los que más dificultades dicen encontrar para hacerlo (por escasez de lugares). Las otras razones importantes argüidas son la falta de costumbre, la falta de tiempo, la falta de información y la comodidad (Ministerio de Medio Ambiente, 2014). En relación al rango etario, el tramo de 55 a 64 años es el que más recicla (30\%) frente a los jóvenes de 15 a 24 años (9\%) y de 25 a 34 años (15\%). Si los jóvenes no reciclan quizás sea porque sus padres lo hacen por ellos o están desmotivados.

Gráfico 4.

Reciclaje según grupo socioeconómico (\%)

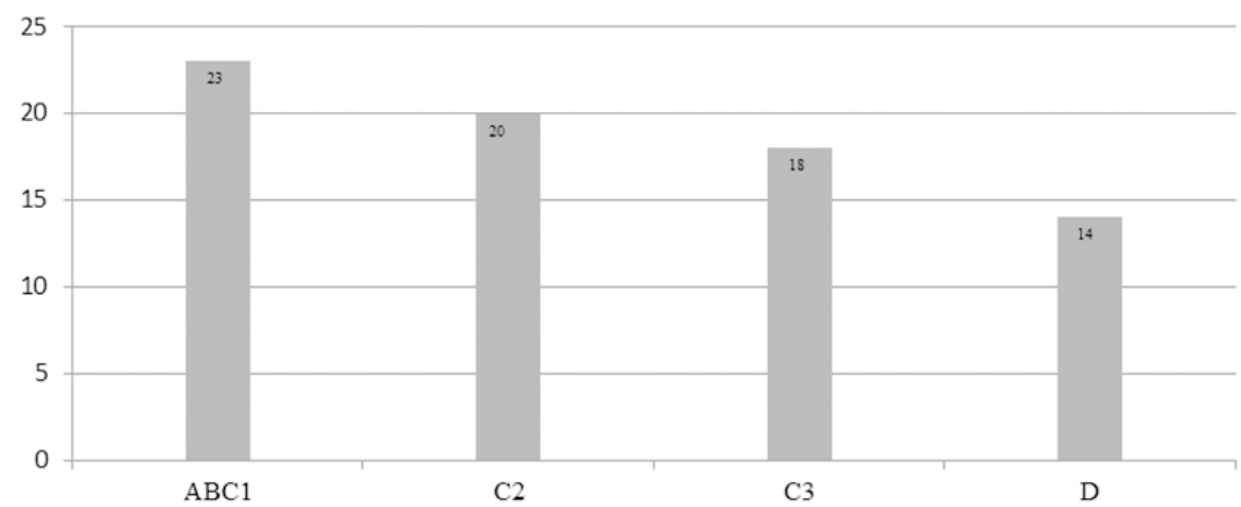

Fuente: Elaboración propia con datos de Adimark (2015) ${ }^{20}$ 
En la Primera Encuesta Nacional de Medio Ambiente (2014), se elaboró un índice de Conductas Ecológicas, ponderando diversas actitudes y acciones ${ }^{21}$ para calificar el grado de preocupación que los ciudadanos tienen por el medio ambiente ${ }^{22}$. A partir de este índice se puede ver ciertos sesgos importantes como los etarios y de nivel socioeconómico. Si bien no es demasiado, la tendencia a tener "conductas ecológicas" aumenta levemente en los sectores de mayores recursos en la población, mientras que, el rango etario más ecológico serían los adultos. Siguiendo con prácticas de cuidado del medio ambiente, también llama la atención las razones para usar la bicicleta según encuesta de la UNAB 2014: solo el 8\% reconoce hacerlo para no dañar el medio ambiente; en su mayoría, responden a otros motivos (distraerse (39\%) hacer ejercicio (22\%), rapidez (15\%) y ahorro (12\%)).

Respecto a la percepción sobre las iniciativas gubernamentales, estas no parecen haber tenido gran impacto desde la percepción de las personas, pues más del $60 \%$ la opinión pública señala que el gobierno no dedica esfuerzos suficientes para cuidar el medio ambiente ni para educar en estos temas en los colegios (Ministerio de Medio Ambiente, 2015). En todo caso, las encuestas de opinión pública muestran una desconfianza generalizada en las instituciones (la última encuesta UDP indica que sólo un 13\% confía en el gobierno, un 4,1\% en el Congreso y un 2,7\% en los partidos políticos (UDP, 2015), lo que dificulta la aprobación y satisfacción con las políticas públicas ${ }^{23}$.

Si bien, como se ha mencionado, la información que entregan estas encuestas tiene importantes limitaciones (escasa continuidad y poca información sobre temas energéticos), de todos modos permiten algunas observaciones interesantes. En primer lugar, se refleja una preocupación no muy definida que se focaliza en torno a casos puntuales sobre temas relevantes de impacto ambiental, más que una posición general o permanente. Así, por ejemplo 85\% de las personas encuestadas indica que proteger el medio ambiente genera ahorros, pero $51 \%$ declara que hacerlo encarece el costo de la vida. Las personas dicen estar interesadas en el medio ambiente, pero no si implica costos significativos.

Además, si bien las personas muestran interés en temas ambientales este interés no parece profundo pues aparece más bien en acciones puntuales y no en acciones más sistemáticas como reciclaje. En países desarrollados el porcentaje de personas que recicla es significativamente mayor. Por ejemplo, en Estados Unidos, más de la mitad de las personas declara reciclar $75 \%$ o más de lo potencialmente reciclable ${ }^{24}$.

En materia específica de energía, se observa que las personas reaccionan frente al debate público, como se pudo observar en el caso de Hidroaysén, pese a que se ponía en juego el tema de costos vs medioambiente. No se observa diferencias muy significativa por sector socioeconómico, aunque los estratos más altos parecen más críticos (aunque la crítica es relativamente generalizada) y los más bajos están más satisfechos con la información disponible.

\section{Estrategias institucionales en Chile: el caso de la eficiencia energética}

En Chile las políticas públicas en temas de eficiencia energética (y más generalmente, sobre sustentabilidad energética), se han centrado principalmente en fortalecer la institucionalidad. Entre estas, se destaca la creación del Ministerio de Energía en el año 2010 con una división de eficiencia energética y la facultad de crear estándares mínimos de eficiencia energética, la de la Agencia chilena de eficiencia energética como institución público-privada en el año 2010, el Plan de acción de eficiencia energética 2020del año 2012, la Agenda de energía del 2014 y los programas de incentivos a empresas y ciudadanos para mayor eficiencia y fomentar las energías renovables.

Como se mencionó, en el período comenzando en 2014 hubo un esfuerzo especial de participación ciudadana en el proceso Energía 2050, a partir de lo cual se elaboró una política y una hoja de ruta. Dentro de ese proceso, la eficiencia energética junto a la educación resultó como uno de los cuatro pilares de la política con el objetivo último de desacoplar el consumo del crecimiento económico.

Chongy Dubois(2011) identifican cuatro barreras para lograr la eficiencia energética: pérdida de comodidad al adoptar medidas de eficiencia energética, problemas en los incentivos, falta de información y los 
costos. Chatterton (2011), por su parte, argumenta que se puede estudiar el uso de la energía desde distintas teorías. Las teorías económicas, por ejemplo, explican las decisiones energéticas de los ciudadanos en función de los incentivos económicos, las teorías psicológicas, por otra parte, señalan que los individuos actúan en respuesta a la información, las teorías sociológicas están orientadas a la forma en que realizamos las "actividades" en que hay consumo energético, mientras que las teorías educacionales argumentan que el uso energético es una habilidad (skill) que se aprende y se desarrolla.

Sea cual sea la aproximación que se tome, la información es la base para la acción. La falta de información ciudadana se ha intentado superar con diversas acciones gubernamentales como las campañas de sensibilización ${ }^{25}$ que buscan generar un cambio de conducta.

En efecto, en el año 2005,se creó el Programa País de Eficiencia Energética (PPEE) ${ }^{26}$ con el propósito fundamental de crear una cultura de eficiencia energética.Las campañas "Ahorra Ahora” y "Únete a la buena energía” se desarrollaron durante la segunda etapa del PPEE, donde Ruchansky et al. (2011) señalan que se buscaba remover las barreras informativas y culturales que impedían desarrollar la eficiencia energética. Estas eran campañas que motivaban la participación ciudadana durante la etapa de implementación de proyectos se sensibilización ciudadana.

La campaña “Ahorra Ahora” del 2008 tenía un enfoque económico (monetario) con recomendaciones para disminuir el consumo energético (ahorro). Se estima que tuvo un impacto positivo ya que se logró reducir la demanda eléctrica ese año, aunque coincide un período de crisis económica importante por lo que es difícil evaluar su impacto específico. Sin embargo, el impacto no fue sostenible en el tiempo pues, siguiendo a Borregard (2008), no significó una sustitución de fuentes de energía y, por tanto, no fue eficiencia energética, sino sólo ahorro.

Por su parte, el mensaje principal de la campaña "Únete a la buena energía” del 2009 era "ahorras dinero, cuidas el medioambiente y generas buena energía en los demás”. Se observa por un lado que la campaña apunta a factores económicos que coinciden (en su efecto final) con valores sociales. Pero, por otro lado, también presenta incentivos valóricos en el eslogan “cuando usas bien la energía algo se enciende en ti”.

La fundación Pro-Humana destacó que la campaña buscara una transformación de las personas, por cuidar un recurso que es de todos, abandonando acciones individualistas. La campaña tuvo alto impacto considerando que un 79\% de personas que recordaban la campaña indicaron al menos una acción de ahorro energético. Estas acciones eran principalmente apagar luces que no se ocupan, cambiar ampolletas convencionales por eficientes y desenchufar equipos electrónicos sin uso.

Un trabajo reciente (2012) del Gabinete del Primer Ministro del Reino Unido, señalaba que una estrategia importante en el cambio de conducta en los individuos frente al tema energético es tratar a la población más como parte de comunidad que como consumidores indicando que "recentre search suggests that engaging individuals as members of a community, rathert an only as consumers of energy, is an important strategy for changingenergy-related behaviours" (Cabinet Office et al, 2012, p.11).

En contraste, en Chile se ha optado de manera preponderante por un enfoque económico individual. Por ejemplo, la AChEE no trabaja el concepto de eficiencia energética porque lo considera demasiado técnico, "lo tratan desde el punto de vista del presupuesto, mayor control, mayor seguridad, reducción de gases contaminantes, ser más amigables con el medio ambiente, y también reducir costos”27 es decir, donde hay varios conceptos individuales para diversos grupos.

De acuerdo a Natalia Arancibia, de la Agencia Chilena de Eficiencia Energética, los ciudadanos no entienden qué es la Eficiencia Energética, lo ven más como una reducción de un costo de operaciones, lo que es un manejo del presupuesto. La AChEE“es una institución público-privada que tiene que responder a una mirada súper privada de resultados versus una mirada pública que es de construir las bases de conocimiento con el fin de que la gente pueda aceptar, recomendar y participar de estos programas, y esto es súper difícil” (Arancibia).Además, para Arancibia 
"la eficiencia energética es muy difícil sin una normativa porque es un tema netamente voluntario por lo que es necesaria una política a corto y largo plazo que satisfaga las expectativas inmediatas de la sociedad y las necesidades de desarrollo a largo plazo que busca la agenda pública”.

Como señala Anita Aguirre, de la División de eficiencia energética del Ministerio de energía, se observa que el concepto "la buena energía" no se logró instalar en la población donde se percibe de manera difusa ${ }^{28}$. Por lo demás, se trabajó un concepto de ahorro, no de eficiencia.

Esto contrasta con la forma en que se ha generado y presentado información desde organizaciones de la sociedad civil, que han logrado "instalar" temas. Por ejemplo, Patagonia sin Represas es una campaña compuesta por un conjunto de organizaciones nacionales e internacionales, que operan formalmente desde el año 2007. Los resultados de esta campaña se sustentaron en la participación ciudadana en la región a través de reuniones, mesas de trabajo y asambleas informativas, con la finalidad de determinar temas relevantes propios de la región. La información no se ejercía de manera unilateral, sino que se esperaba una retroalimentación por parte de los ciudadanos.

Como medios de difusión de información utilizaron todos los que estuvieron a su alcance (televisión, radio, diario, etc.), pero destacan el uso de las redes sociales en vista de que eran más rápidas y gratis. Además, se sumaron parlamentarios y ciertos sectores empresariales, por lo que el movimiento tuvo más éxito que las campañas de ONGs medioambientalistas de los años noventa contra mega proyectos forestales e hidroeléctricas como la del alto BíoBío de Endesa.

Para Francisco Urquiza, Coordinador general de la oficina de sustentabilidad de la Pontificia Universidad Católica de Chile,

“en Chile no hay nada que califique realmente como una campaña sistemática del gobierno que busque realmente cambiar hábitos. Más bien es a través de la misma ciudadanía organizada y viralmente vía redes sociales que se han ido instalando mensajes y marcando estilos de vida alternativos que comienzan a arraigarse en nuestra sociedad"29.

Según él, las organizaciones sociales han tenido una influencia importante en las nuevas actividades con objetivo ambiental como el uso de la bicicleta, el reciclaje, las energías limpias o la protección de la biodiversidad mientras que las campañas que promueven intereses privados generan el riesgo de mensajes contaminados con el consumismo ${ }^{30}$.

El mensaje de la campaña Ahorra Ahora se dirigía a los ciudadanos como “consumidores” de energía, mientras que el mensaje y discurso de Patagonia sin Represas se dirigía a los ciudadanos como "comunidad" tal como lo hizo la campaña "Únete a la buena energía”. Aunque Patagonia sin Represas y Únete a la buena energía hayan apelado a la "comunidad", no fueron igualmente exitosas en parte porque se trata de problemas de muy distintos y con distintas implicancias. Por lo tanto, la orientación pública o privada de la información no es lo único determinante para los resultados.

En concordancia con la nueva Ley de Participación Ciudadana (ley 20.500) del año 2011, como ya se ha señalado, en todos los ministerios se puso en marcha mecanismos de información (relación unidireccional) y consulta (relación bidireccional) entre las instituciones públicas y los ciudadanos. Se han creado los Comités Consultivos Ciudadanos para formar grupos temáticos de discusión con un público más general y se mantienen las comisiones de expertos seleccionados.

En el caso del Ministerio de energía se creó la División de participación y diálogo social en el año 2014 con el objetivo de "ciudadanizar la información y el leguaje de la energía en Chile”31. Sin embargo, para Natalia Arancibia, Subdirectora de la AChEE, "el ciudadano no ha visto al Ministerio de Energía como un organismo cercano para dar respuesta, sólo para las organizaciones de expertos (empresas del sector, ONG, etc.)”32 quizás porque hay otros organismos que aparecen más involucrados en la actividad más cotidiana como el Ministerio del medio ambiente o la misma AChEE. 
Para Marcelo Padilla, de la División de eficiencia energética del ministerio de energía ${ }^{33}$, esta falta de confianza aparece en algunos talleres ya que "muchas ONG de la sociedad civil como Greenpeace se quejan de que el discurso de eficiencia energética del Ministerio de energía se contradice con la aprobación gubernamental de megaproyectos de energía que no cuidan el medio ambiente”. En este contexto, el gran interrogante para el funcionario de este ministerio es cómo poder canalizar las expectativas ciudadanas que se han venido creando con la Agenda de Energía si no hay confianza en el Ministerio de energía ${ }^{34}$.

De todos modos, el Estado chileno ha dado otros pasos para la participación ciudadana. Por ejemplo, un hito fue la creación de una división de participación y diálogo social en el Ministerio de energía. Previamente, el Ministerio "no tenía trato con la ciudadanía de forma directa”35, sino que trabajaba con las empresas generadoras, transmisoras y distribuidoras de energía y se dejaban los temas medioambientales para el Ministerio de Medio Ambiente el cual respondía a las inquietudes ciudadanas. Como explica Javier Zulueta, Jefe de la Unidad de Participación y Diálogo del Ministerio de Energía, se busca “ciudadanizar” el lenguaje de la energía en Chile, ya que los conceptos técnicos son poco comprensibles en la ciudadanía lo que no fomenta la participación $^{36}$.

La nueva Agenda de energía del gobierno ha buscado nuevas formas de participación en la formulación de políticas públicas hasta ahora limitadas a grupos de expertos. La estrategia es más integral, buscando también la formación cívica desde los colegios para "formar una opinión crítica sobre el sector energía y cambiar la cultura de participación”37. En eficiencia energética la AChEE participa en todos los procesos de sensibilización, incluyendo los proyectos de educación primaria ${ }^{38}$, y también han coordinado con 22 universidades reducir el consumo energético tanto en el campus universitario como en la conducta de la comunidad académica a través de un nuevo diseño de mallas curriculares que incluyan la buena gestión de la energía ${ }^{39}$.

Uno de los mayores impulsos fue "Energía 2050"40, el proceso participativo estuvo compuesto de tres grupos: Comité consultivo (nivel político), grupos expertos de trabajo (nivel técnico) y finalmente la ciudadanía a través de una plataforma multimedia y talleres regionales (nivel social) pero en la que participan siempre los mismos expertos.

Los resultados de participación fueron acotados. Por ejemplo, en la consulta sobre la propuesta de la política energética 2050 se recibieron más de cien consultas y propuestas que fueron contestadas por la administración, aunque no hubo debate, sino más bien respuestas técnicas ${ }^{41}$. También se puso en marcha cuentas anuales en las que se explica la gestión ministerial de una manera transparente y participativa. Para el Ministerio de energía la primera se realizó en marzo de 2015, pero en rasgos generales la participación ciudadana sigue estando limitada a los funcionarios y expertos del mundo académico y empresarial.

Como mencionamos anteriormente, para algunos autores estos mecanismos de consulta se reducen a una participación “simbólica” (Arnstein, 1969) o de “fachada” (Prieto- Martín, 2010).

Esta participación "limitada” no es exclusiva del sector energía. En proyectos en general hay una falta de participación. En el sistema de evaluación de impacto ambiental ${ }^{42}$, los procesos son demasiado técnicos para la comprensión ciudadana, cortos para la recopilación de comentarios y firmas y no son vinculantes (Tironi, 2011; Dufey, 2013). Para estos autores, la participación deber ser temprana (desde la formulación del proyecto), con metodologías deliberativas como foros y mesas y con procesos de votación local, contando con una adecuada información y ayudas para financiar los gastos de participación de los grupos afectados.

Ha habido experiencias de participación ciudadana a través de la evaluación ambiental estratégica $^{43}$ (EAE) desde el año 2011 (aunque sólo es una exigencia legal desde 2015) para debatir los cambios en políticas, planes y programas públicos en cualquiera de los tres niveles de gobierno, conjuntamente los Ministerios de Energía, Medio Ambiente y Bienes Nacionales. No obstante, aún se reciben críticas respecto de que siempre participan las mismas personas en las mesas y que éstas suelen ser “expertos” y no el “ciudadano afectado" 44 . 


\section{Conclusiones}

La incorporación de la opinión ciudadana en las definiciones de políticas públicas aparece como una reivindicación importante de los procesos democráticos modernos. Sin embargo, lograr esto de manera real y efectiva no resulta un proceso sencillo y simple, sino como un proceso en continua evolución.

Desde el comienzo de las políticas de eficiencia energética se incorporó la información como un aspecto relevante de la política, pero básicamente como forma de generar cambios de hábitos en consumo energético definidos en la política. En esa lógica, se establecieron mecanismos solo unidireccionales de información (desde el Estado hacia el ciudadano) para transmitir una política y no para generar antecedentes relevantes para ésta. Como indica Arnstein (1969) estos intentos de "educar la ciudadanía” para buscar su apoyo son ilusorios y no-participativos, ya que la gente tiene poco influencia y oportunidades para expresarse.

El primer esfuerzo formal de recoger información para la política energética fue el proceso Energía 2050, cuyo objetivo expreso era desarrollar una política energética participativa ("un proceso histórico por su forma participativa de construir una política energética de Estado”45). Se crearon mesas de trabajo para recibir información de los ciudadanos. Sin embargo, además de la dificultad que existe para tener una participación amplia por estos medios, el procesamiento de los resultados de las mesas recayó en un Consejo Consultivo, formado por "expertos” y no pasaba directamente al debate político.

De ese modo, se reprodujo en buena medida los mecanismos de participación que se han llevado adelante en otros ámbitos (como pensiones, trabajo, infancia y calidad de la educación), recogiendo información de la sociedad civil en un esquema poco claro, para encargar finalmente a un grupo de expertos las recomendaciones que serán sometidas a debate. De ese modo, tampoco se motiva al público general (no experto) a participar masivamente. Además, la complejidad de la participación sugiere que participan aquellos que pueden tener más facilidades para hacerlo y no necesariamente hay una representación adecuada de intereses.

Adicionalmente, no se ha puesto énfasis suficiente en una participación ciudadana bien respaldada técnicamente, con el propósito de que los ciudadanos puedan participar más directamente de estos procesos formando una opinión crítica sobre el sector de la energía. Hasta ahora los mecanismos de información se centran sobre todo en información digital, en la creación de información escolar y, en menor medida, en campañas esporádicas con un alto componente técnico, lo que no está pensado como sensibilización para la participación ciudadana a largo plazo sino a una participación coyuntural.

La información entregada no buscó este desarrollo de ciudadanos activos y, de hecho, como se ha señalado, se enfoca en una lógica más mercantil, enfatizando principalmente el beneficio propio de ciertas acciones. No se tiene una lógica ciudadana que lleve a la reflexión sobre los objetivos de eficiencia energética y desarrollo en el largo plazo que pueda involucrar otros valores (en particular los valores postmaterialistas) en políticas globales, sino que busca resultados concretos.

Quizás la contrapartida de esto ha sido un cierto populismo en proyectos específicos, sin alterar las grandes políticas. Así, por ejemplo, se puede entender la reacción de las autoridades frente a las protestas en ciertos casos. Son emblemáticos de esta situación lo ocurrido en Barrancones (donde ante protestas masivas en la capital frente a un proyecto termoeléctrico de 2000MW en una zona costera del norte de Chile, el Presidente de la República se comunicó con los altos directivos de la empresa para solicitar modificar la iniciativa, que ya tenías las aprobaciones correspondientes) y lo ocurrido frente al proyecto Hidroaysén (donde finalmente las autoridades Ministeriales decidieron no aprobar el proyecto de centrales hidroeléctricas de casi 3000MW en el extremo sur de Chile, tras una progresiva opinión negativa en las encuestas.

En síntesis, la opinión ciudadana no sería tomada en cuenta en lo relevante para políticas, pero podría tener influencia en proyectos específicos. De hecho, para la formulación de políticas públicas, la opinión de los ciudadanos parece ser un antecedente lejano. Las encuestas de opinión sugieren que no hay una sintonía con las políticas públicas ya que éstas no tienen en cuenta los intereses emergentes. Por ejemplo, la opinión de los ciudadanos sobre la preeminencia de la protección del medio ambiente incluso a expensas del crecimiento no 
parece haber sido tomada en cuenta en absoluto. Desde el sector público, prevalece un enfoque en lo económico (ahorro económico por ejemplo, cuando se trata de eficiencia energética) y el mercado.

En parte, el problema puede tener su origen en el carácter algo indefinido de la opinión pública en el tema energético cuando se trata de sustentabilidad, pero es difícil también tener más análisis cuando las encuestas de opinión no tienen continuidad en el tiempo ni la profundidad necesaria. El carácter voluble de la opinión pública podría estar marcando una verdadera indefinición en valores o, sencillamente, una contradicción entre valores y condiciones materiales. Eso también requiere una exploración más profunda. En ese sentido, sería de gran valor tener una encuesta de opinión especializada en temas de energía y sustentabilidad que entregue información regular y comparable.

No obstante, lo que hemos señalado respecto a la opinión pública no quedará adecuadamente reflejado sin la presencia de ciudadanos suficientemente activos y preparados como para representar los diversos intereses adecuadamente en las políticas públicas y sin los mecanismos institucionales para generar un diálogo en la ciudadanía respecto a diversas opciones de política.

Por ejemplo la AChEE no tiene ni la competencia ni los recursos para hacer consultas ni para evaluar la participación ciudadana. De acuerdo a Arancibia "para que le entregue un presupuesto para participación y entender bien cómo funciona el "no experto" requiere también un cambio de mentalidad y un cambio de lo que se espera de la agencia (Arancibia, 2014).

Hasta ahora los expertos de las mesas de trabajo coinciden en que la "Agencia de Eficiencia Energética tiene el peor de los mundos, lo peor de lo privado y lo peor de lo público. La energía en tema social finalmente, si uno no lo entiende como un tema social va a quedar en un círculo técnico” (Padilla, 2014).

En ese sentido, los cambios institucionales son fundamentales para dar respuesta a una exigencia de sociedad más participativa. Falta mejorar las instituciones de tal manera de generar una participación activa y una cultura de participación con resultados duraderos. La poca claridad del impacto de la participación en las políticas puede afectar la participación misma. Ejemplos en otros países de la OCDE como las conferencias de búsqueda de consenso o los jurados ciudadanos, son opciones relevantes a considerar ${ }^{46}$. Se trata no sólo de recibir información, consultar o recibir comentarios, sino de generar diálogos que puedan aportar a mejores políticas.

Las campañas mostradas en este trabajo relativas a eficiencia energética, revelan que lo que se busca son objetivos puntuales, informar o crear consciencia sobre ciertos temas, para que los ciudadanos "entiendan" algo, pero rara vez se trata de involucrar y entregar información que promueva y facilite una verdadera participación que genere una búsqueda colectiva de eficiencia energética.

Es decir, la información debería estar orientada a la sensibilización para cambiar conductas hacia aquellas que parezcan más relevantes para la población y no a la mera entrega de información. Esto sugiere la importancia de usar otras teorías para el diseño de estas políticas, tales como la educacional, sociológica o psicológica mencionadas por Chatterton (2011), y no sólo enfocarse en las teorías económicas de la decisión racional con el propósito de llegar verdaderamente a los ciudadanos. Los incentivos económicos parecen motivar a corto plazo, pero no implican un cambio sustentable.

Como indica Annie Dufey (2014), "la participación es un proceso en construcción y el desafío para el Ministerio es crear esa cultura de participación de manera interna, como una herramienta inserta en la toma de decisiones”. Esto permitiría acercar la política pública al ciudadano y generar más confianza en las instituciones, pues la desconfianza mantendrá a las personas alejadas de la participación.

En síntesis, los estudios de opinión pública sugieren que hay intereses y visiones distintas presentes en la ciudadanía respecto a la eficiencia energética que la que se revela en las políticas públicas. Por otra parte, no hay manera de verificar cuáles podrían ser ni cómo contrastar diferentes visiones porque no hay mecanismos de participación ciudadana adecuados para ello. Además, las estrategias de información para mejorar la eficiencia 
energética en los últimos años, no conducen a una participación activa y relevante que incorporen todas las visiones a las decisiones finales en políticas públicas. Los ciudadanos recurren a las protestas en la calle y al debate público en las redes sociales para que haya cambios en las políticas pero no es una participación activa sino una expresión de sus opiniones que no necesariamente tienen eco en el proceso de toma de decisiones políticas.

En consecuencia, es difícil que se logre el desarrollo de políticas de eficiencia energética adecuadas a los desafíos actuales en el sector energía, si no hay cambios significativos en todo el proceso de participación.

\section{Agradecimientos}

Agradecemos la colaboración de Bárbara Callejas y Sebastián Estay en la recopilación y proceso de datos y entrevistas para la elaboración de este trabajo de investigación.

\section{Nota}

${ }^{1}$ Los sistemas de información deberían ser capaces de sensibilizar a todos los ciudadanos de una manera incluyente para que la eventual participación sea realmente relevante para las políticas Así, por ejemplo, no se debería enfatizar los foros en las redes sociales en los que los jóvenes aparecen como el público objetivo (Castells, 2009; Valenzuela, 2011). Las redes sociales (vía Internet) constituirían más bien un espacio de “subactivismo" que ayuda a la conformación de identidades y de base para la actividad política o ciudadana, pero en muchos casos no constituyen acción política como tal (Arriagada et al, 2012).

${ }^{2}$ De acuerdo al Instituto para la Planificación Efectiva para prevenir y resolver conflictos medioambientales "la información debe estar disponible libremente para todos los participantes" (Institute for Participatory Planning, 1981). En: Edward, C. 2008, p.45.

${ }^{3}$ https://www.un.org/spanish/esa/sustdev/agenda21/riodeclaration.htm

${ }^{4}$ https://www.unece.org/env/pp/treatytext.html

${ }^{5}$ http://www.energia2050.cl/es/energia-2050/descripcion-del-proceso/

${ }^{6}$ La Agencia Chilena de Eficiencia Energética (www.acee.cl), señala que el "uso eficiente de la energía es reducir la cantidad de energía eléctrica y de combustibles que utilizamos, pero conservando la calidad y el acceso a bienes y servicios. Usualmente dicha reducción en el consumo de energía se asocia a un cambio tecnológico (aunque) puede estar vinculada a una mejor gestión o cambios en los hábitos y actitudes”.

La AChEE distingue eficiencia de ahorro, pues "ahorrar energía (...) puede significar reducir o dejar de realizar determinadas actividades, para evitar el consumo de energía”. Como ejemplo, señala lo siguiente: "el ahorro energético se genera cuando apagamos la luz para reducir el consumo de energía. Si, en cambio, reemplazamos la ampolleta incandescente por una eficiente, estamos tomando una medida de eficiencia energética, que nos proporcionará una disminución en el consumo de energía, sin perjuicio a desarrollo de nuestras actividades”.

${ }^{7}$ Directive 2003/35/EC de la UE establece la obligatoriedad de la participación ciudadana en en la preparación. modificación o revisión de planes y programas medioambientales y Public Involment Policy de la Agencia norteamericana de Protección Ambiental propone consultas públicas y otras actividades de participación.

${ }^{8}$ En la elaboración abierta de Políticas se consideran plataformas web y redes sociales, “crowdsourcing” y laboratorios de política. House of Commons Public Administration Select Committee Public engagement in policy-making Second Report of 
Session 2013-14 . https://publications.parliament.uk/pa/cm201314/cmselect/cmpubadm/75/75.pdf (consultado 22.04.19)

${ }^{9}$ La Ley 20500 de 2011, sobre Asociaciones y Participación Ciudadana en la Gestión Pública, marcó un hito al introducir la participación como un derecho. El artículo 69 establece que el "Estado reconoce a las personas el derecho de participar en sus políticas, planes, programas y acciones”.

${ }^{10}$ En el caso de Chile, un ejemplo podrían ser las campañas de Patagonia sin Represas en contra de Hidroaysén, ya que los incentivos ciudadanos para participar se enfocaban en términos valóricos y no materiales, de protección al medio ambiente.

${ }^{11}$ La falta de consistencia puede también incidir en los niveles de información y por tanto de participación.

${ }^{12}$ Encuesta telefónica (metodología CATI), en las capitales regionales (15) de Chile a una muestra de 5.057 personas mayores de 18 años con teléfono fijo o celular, entre octubre y diciembre de 2014.

${ }^{13}$ La pregunta fue ¿Está usted a favor o en contra de la construcción de las centrales hidroeléctricas en la Región de Aysén? Encuesta realizada entre 11 de marzo y 3 de abril de 2011, por medio de entrevistas telefónicas 1.024 (entre hombres y mujeres) mayores de 18 años residentes de 24 ciudades con más de 50 mil habitantes de Arica a Punta Arenas.

${ }^{14}$ Por ejemplo, el 20 de mayo de 2011 casi 70.000 personas se manifestaron frente a La Moneda (INDH,

${ }^{15}$ De acuerdo a la Asociación de Investigadores de Mercado los grupos socioeconómicos en Chile serían: Clase alta (AB), clases media-alta, emergente y baja (C1-C2-C3), vulnerable (D) y pobre (E).

http://www.aimchile.cl/wp-content/uploads/Presentaci\%C3\%B3n-final-AIM.pdf

${ }^{16}$ En general no más del 28\% de los encuestados estaría dispuesto a comprar productos ecológicos que sean un 20\% más caro (Encuesta UNAB IPSOS 2012).

${ }^{17}$ La pregunta fue: ¿cuánto más pagaría por un producto que cuide el medio ambiente? Resultados obtenidos de 804 entrevistas vía web a hombres y mujeres mayores de 18 años residentes de Santiago y regiones entre el 10 y el 29 de enero de 2013.

${ }^{18}$ La pregunta fue: ¿con cuál de estas afirmaciones sobre el medio ambiente y la economía está usted más de acuerdo? Entrevista presencial a 1.302 personas hombres y mujeres mayores de 18 años residentes en 89 comunas del país realizada entre los días 22 de septiembre y 20 de octubre de 2014.

${ }^{19}$ Según Valenzuela et al (2012), los movimientos estudiantiles y ambientalistas chilenos no solo usan las protestas como medio de acción. Se han caracterizado por incluir otras formas de acción política directa, tales como firmar peticiones, participar en debates universitarios, enviar cartas a los medios de comunicación y reunirse con autoridades locales y de gobierno.

${ }^{20}$ La pregunta fue: ¿usted recicla? Entrevistas cara a cara en hogares a 3.200 personas (hombres y mujeres), mayores de 15 años, de los segmentos $\mathrm{ABC} 1$ hasta el $\mathrm{D}$, de todas las capitales regionales, más las ciudades de más de 100 mil habitantes representando un 80\% de la población urbana de Chile.

${ }^{21}$ Por ejemplo: separar basura, comprar con bolsas de tela, usar baterías recargables, apagar luces.

${ }^{22}$ Según Hajek (1993), para medir la conciencia ambiental en Chile se debe revisar las acciones de gobierno, en cuanto a la institucionalidad, a la educación, a los empresarios, a los grupos de acción ecológica y a los medios de prensa para saber cómo éstos han ido reaccionando a los desafíos de la protección del medio 
ambiente. Si existen cambios significativos con repercusiones notorias se podría hablar de una incipiente conciencia ambiental.

${ }^{23}$ Por otra parte, como afirman Chong y Dubois (2011), es probable que la información que proviene del entorno social de los individuos se perciba con mayor credibilidad, por lo que es normal que existan muchas críticas a las acciones (o inacción) del gobierno.

${ }^{24}$ https://www.americanchemistry.com/Media/PressReleasesTranscripts/ACC-news-releases/NewSurvey-Half-of-Americans-Say-They-Recycle-75-Percent-or-More-of-Recyclable-Items.html

${ }^{25}$ Se entiende por sensibilización el "proceso mediante el cual la población (o incluso una persona) conoce un tema y se apropia de él, siendo consciente de sus características, la problemática que hay detrás y cómo se podría actuar al respecto” (Pardo, 2006:10).

${ }^{26}$ Su continuidad actual es la Agencia Chilena de Eficiencia Energética (AChEE). Ruchansky et al. (2011) señalan que una de las aspiraciones de Chile para el año 2005 era formar parte de la Organización para la Cooperación y el Desarrollo Económico (OCDE), para lo cual el país se sometió a una evaluación de desempeño de impacto ambiental realizada por la OCDE y CEPAL que llevó, entre otras cosas, a la formación del PPEE.

${ }^{27}$ Entrevista a Natalia Arancibia, Subdirectora de la Agencia Chilena de la Eficiencia Energética (AChEE), 11 de noviembre 2014.

${ }^{28}$ Entrevista a Anita Aguirre, División de Eficiencia Energética, Ministerio de Energía. 11 de noviembre 2014

${ }^{29}$ Entrevista a Francisco Urquiza, Coordinador general de sustentabilidad UC (4 noviembre 2015) http:/ /sustentable.uc.cl/

${ }^{30}$ Ibid.

${ }^{31}$ La creación de esta División permite sistematizar, generar, guiar distintos espacios de participación a nivel del Ministerio. El Ministerio por primera vez cuenta con una oficina de información al público por lo que es cada día más cercano a los ciudadanos.

${ }^{32}$ Entrevista a Natalia Arancibia subdirectora de AChEE, 11 de noviembre de 2014.

${ }^{33}$ Entrevista a Marcelo Padilla, 7 noviembre 2014.

${ }^{34}$ La Agenda de la Energía ha sido el primer hito participativo del Ministerio de Energía en el proceso de Energía 2050. http://www.energia2050.cl/programa/index/

${ }^{35}$ Entrevista a Javier Zulueta, Jefe de la Unidad de Participación y Diálogo del Ministerio de Energía, 7 noviembre 2014.

${ }^{36}$ Ibid

${ }^{37}$ Entrevista a Annie Dufey. Jefe División de Prospectiva y Política Energética, Ministerio de Energía, 7 de noviembre de 2014. Ver también el portal Educar Chile. http://www.educarchile.cl/ech/pro/app/home

${ }^{38}$ Entre los programas de Educación Ciudadana busca la instauración de la EE en actividades docentes y proyectos educativos desde la educación pre-escolar a la superior. La página web busca promover la participación en la EE de diversos sectores de la sociedad a través de un lenguaje simple, se realizan juegos interactivos, programas de televisión, etc. www.educachee.cl 
${ }^{39}$ Entrevista a Natalia Arancibia. Subdirectora de la Agencia Chilena de Eficiencia Energética (AChEE) el 11 noviembre 2014.

${ }^{40}$ Energía 2050 es un proceso participativo de tipo consultivo para la creación de una política energética de largo plazo con una visión social, política y técnica compartida. El proceso participativo está compuesto de tres grupos: Comité Consultivo (nivel político), Grupos Expertos de Trabajo (nivel técnico) y finalmente la ciudadanía a través de una plataforma multimedia y talleres regionales (nivel social). La información que entrega la plataforma actualmente consiste en avances en el proceso participativo, información sobre Energía 2050, avances de las mesas de trabajo, agenda de energía, calendario de trabajo, entre otros. Por otro lado, los talleres regionales son encuentros regionales que trabajan en mesas temáticas seleccionadas. Actualmente se han realizado en Antofagasta, Puerto Montt, por nombrar algunos. http://www.energia2050.cl/

${ }^{41}$ La plataforma virtual de Energía 2050 dispone de información relevante del proceso: objetivos, descripción, plazos, insumos, formas de participar y comentarios. En dicha plataforma se dispone de toda la información del trabajo de los GET con la finalidad de que los ciudadanos reaccionen y comenten. La información que entrega la plataforma actualmente consiste en avances en el proceso participativo, información sobre Energía 2050, avances de las mesas de trabajo, agenda de energía, calendario de trabajo, etc.

${ }^{42}$ La Evaluación de Impacto Ambiental es obligatoria para la mayor parte de los proyectos energéticos.

${ }^{43}$ Entre las características de este instrumento se destaca el hecho de que obliga al promotor de la EAE en curso (por ejemplo, el municipio, un ministerio o un gobierno regional) a interactuar con otros organismos públicos para integrar sus visiones. También contempla la consulta ciudadana. http://portal.mma.gob.cl/ evaluacion-ambiental-estrategica/

${ }^{44}$ Entrevista a Dufey y Padilla 2014.

${ }^{45} \mathrm{http}: / /$ www.energia2050.cl/programa

${ }^{46}$ En las conferencias de búsqueda de consensos, un grupo aleatorio de 10 a 15 ciudadanos no expertos discuten y presentan sus conclusiones consensuadas (se usa frecuentemente en países como Dinamarca o Noruega), mientras que los jurados ciudadanostienen la forma de un tribunal abierto al público en el que se interroga a los expertos y las conclusiones no tienen que consensuarse, como en el caso francés (OCDE, 2006).

\section{Bibliografía}

Abaza, H. et al (2004). Enviromental Impact Assessmet and strategic Environmental Assessment: Towards an Integrated Approach. United Nations Environemtal Programme. https:/unep.ch/etu/publications/ textONUBr.pdf

Agencia Internacional de Energía. (2015a). Energy Efficiency Market Report 2015: Market Trends and MediumTerm Prospects. Paris: Agencia Internacional de Energía.

Agencia Internacional de Energía. (2015b).Indicadores de Eficiencia Energética: Bases Esenciales para el Establecimiento de Políticas. Paris: Agencia Internacional de Energía

Aldunate, C., et al. (2011). Jóvenes, participación y medios: Encuesta jóvenes, participación y consumo de medios, Periodismo UDP- Feedback. Santiago de Chile: Centro de Investigación y Publicaciones Facultad de Comunicación y Letras Universidad Diego Portales

Arnstein, S. (1969).A Ladder of Participation, Journal of the American Plannig Association,(35), No. 4,216-224. 
Arriagada, A., et al.(2012).The social media basis of youth protest behavior:The case of Chile, Journal of Communication, (62), Núm2,abril,299-314

Asociación de Investigadores de Mercado (AIM). Recuperado: http://www.aimchile.cl/wp-content/uploads/ Presentaci\%C3\%B3n-final-AIM.pdf

Borregaard, N. (2008). Eficiencia energética en Chile, Menos es Más, Observatorio Económico, N²7, diciembre, 1-8.

Bowen, S., Fábrega, F., y Medel., R. (2012). Movimientos Sociales Rurales y Problemática Medioambiental: La disputa por la territorialidad, Psicoperspectivas. Individuo y Sociedad, (11), No. 1, enero-junio, 204-225.

Cabinet Office. (2011). Behaviour Change and Energy Use. London: Cabinet Office Behavioural Insights Team.

Castells, M. (2009).Comunicación y poder. Madrid: Alianza.

_ M.(2010). “Comunicación y Poder en la Sociedad Red”. Conferencia dictada en la Cátedra Democracia y Globalización de la Universidad Diego Portales, 23 junio 2010, Santiago de Chile.

Disponible en: http:/globalizacionydemocracia.udp.cl/wpcontent/uploads/2014/03/ MANUEL_CASTELLS_2010.pdf

CEPAL, \& OIJ. (2007). La juventud en Iberoamérica: Tendencias y urgencias (2a ed.). Buenos Aires: CEPAL, NacionesUnidas.

Chatterton, T. (2011). An introduction to Thinking about "Energy Behaviour": A Multi Model Approach. Londres: Departament of energy \& Climate Change.

Chong, E., y Dubois, U. (2011). Household vulnerability and energy conservation behavior.Paris: ADIS, Université Paris-Sud 11 \& ISG.

Couso, J. (2004). Consolidación Democrática y Poder Judicial: Los Riesgos de la Judicialización de la Política, Revista de Ciencia Política, (24), Núm 2, 29-48

Downs, A. (1957). An economic theory of political action in a democracy, Journal of Political Economy, (65), Núm 2, 135-150

Dufey, A. (2013). Participación ciudadana en el sector energético chileno: Elementos clave y propuestas (Documento de referencia $\left.\mathrm{N}^{\circ} 18\right)$. Santiago de Chile: EspacioPúblico.

Edward, C. (2008). Finding Solutions for Enviromental Conflicts. Power and Negotiaton.Northhampton: Edward Elgar.

Fuenzalida., M. y Quiroz., R. (2012). La dimensión espacial de los conflictos ambientales en Chile, PolisRevista Latinoamericana, (11), No31, abril, 157-168. Recuperado https://scielo.conicyt.cl/ scielo.php?script=sci_arttext\&pid=S0718-65682012000100009

Goodin, R., Moran, M., y Rein, M. (2006). The Public and its Policies. En Goodin et al (Eds).The Oxford Handbook of Public Policy.Nueva York: Oxford University Press.

Guiloff, M. (2011). Nueva institucionalidad ambiental: Hacia una regulación deliberativa. En Couso, J (Eds). Anuario de Derecho Público. Santiago de Chile: Ediciones UDP.

Hajek, E. (1993). La conciencia ambiental en Chile, Contribuciones, (1), Núm93, 35-44 
Halpern, D. (2013). Primer informe abril 2013: Activismo medioambiental 2.0. Observatorio de redes sociales Tren digital, Facultad de comunicaciones UC y consultora medio ambiental Jaime Illanes y asociados

Inglehart, R. (1990). Culture Shift. Nueva Jersey: Princeton University Press

Instituto Nacional de Derechos Humanos (INDH). (2013). Mapa de los conflictos medioambientales Recuperado de: http://www.indh.cl/mapaconflictos/

Martín-Barbero, J. (1992). Pensar la sociedad desde la comunicación. Un lugar estratégico para el debate a la modernidad, DIÁLOGOS de la Comunicación, Núm 32, marzo. Recuperado http://www.dialogosfelafacs.net/ wp-content/uploads/2012/01/74-revista-dialogos-pensar-la-sociedad-desde-la-comunicacion.pdf

Mc Phee, B. (2010). Conflictos ambientales y respuestas sociales: el caso de reetnificación de la comunidad de Quillagua, Revista MAD, No22, mayo, 42-55

Ley N²0.500 Sobre Asociaciones y Participación Ciudadana en la Gestión Pública. (2011). Santiago de Chile: Ministerio Secretaría General de Gobierno. Recuperado http://www.leychile.cl/ Navegar?idNorma $=1023143$

Montero, J. (2011). El medio en los medios: Teoría y práctica del periodismo ambiental (Manual n¹). Agencia de la Energía, Ayuntamiento de Sevilla.

OCDE. (2006). Manual de la OCDE sobre información, consulta y participación en la elaboración de Políticas Públicas. Paris: OECD Publishing

OCDE. (2013). Science, technology and industry scoreboard: Innovation for growth. Paris: OECD Publishing.

Oficina Ministerial de Atención a la Ciudadanía, Subsecretaría Ministerio de Energía Consulta

AU002W-0000937, 24 febrero 2015

Patagonia sin Represas. Recuperado:http://www.patagoniasinrepresas.cl/final/quienes-somos.php

Pardo, C. (2006). Sensibilización ciudadana y cambio de comportamiento en transporte sostenible: Curso de entrenamiento, 2da edición (División 44, Medioambiente e infraestructura). Bogotá: Deutsche Gesellschaft für Technische Zusammenarbeit (GTZ)

Price, V. (1992). La Opinión Pública. Paidós, Barcelona.

Prieto-Martin, P. (2010). Las alas de Leo: la participación ciudadana en el siglo XX. Panajachel Bulbok.

Prohumana. (2009). Campaña de Ahorro Energético que se enciende en ti. Recuperado: http://prohumana.cl/2009/ 10/nueva-campana-de-ahorro-energetico-que-se-enciende-en-ti/

Programa de las Naciones Unidas para el Desarrollo (2008). Informe sobre Desarrollo Humano 2007-2008: “La lucha contra el cambio climático: Solidaridad frente a un mundo dividido”. Programa de las Naciones Unidas para el Desarrollo

Programa País Eficiencia Energética. (2012).Guía Práctica de la Eficiencia Energética. Fundación Chile y Dirección Chile Compra.

Raynes-Goldie, K., \& Walker, L. (2008). Our space: Online civic engagement tools for youth. En W. L. Bennett. (Ed.). Civic life online: Learning how digital media can engage youth .Cambridge, MA: The MIT Press. 161-188. 
Rau, I., Schweizer-Ries, P., y Hildebrand, J. (2012). Participation: the silver bullet for acceptance of renewable energies?.En Kabish, S., Schweizer-Ries, P., y Steinfuhrer, A. (eds). Vulnerability, Risks, and Complexity. Impacts of Global Change on Human Habitats.Hogrefe: Gottingen.

Rau, I. (2013). Presentación en el Seminario Internacional “Energía y Ciudadanía”. Universidad Diego Portales, ICSO y CEDS. 16 noviembre, Santiago de Chile.

Rojas, H., y Pérez, I. (Eds.). (2009). Comunicación y participación política. Bogotá:Universidad Externado de Colombia.

Rodríguez, N., Rudnick, H., Tironi, M. y Varas, P. (2013). Latin America Goes Electric,IEEE Power and Energy Magazine, (11), Núm 3, mayo-junio, 66-75

Ruchansky, B., de Buen, O., Januzzi, G., y Romero, A. (2011). Eficacia institucional de los programas nacionales de eficiencia energética: Los casos del Brasil, Chile, México y Uruguay (En CEPAL Serie Recursos Naturales e infraestructura, $N^{\circ}$ 152). Santiago de Chile: Naciones Unidas.

Sierra, L. (2008). Reforma de la institucionalidad ambiental y oportunidades, Estudios Públicos, Núm 111, 57102.

Tironi, M. (2011). Análisis de las tendencias de opinión y percepciones de la sociedad sobre desarrollo energético y medio ambiente. Paper sin publicar realizado en el marco del trabajo desarrollado por la Comisión Asesora para el Desarrollo Eléctrico (CADE).

Valenzuela, S. (2011).Unpacking the Use of Social Media for Protest Behavior. The Roles of Information, Opinion Expression, and Activism. American Behavioral Scientist, (57), Núm 7, 920-942.

Valenzuela, S. et al. (2012),La protesta en la era del Facebook: Manifestaciones juveniles y uso de redes sociales en Chile 2009-2011”.En Scherman, A. (Eds.). Jóvenes, participación y medios 2011: Encuesta jóvenes, participación y consumo de medios. Santiago de Chile: Periodismo UDP; Feedback. Recuperado: http:// cip.udp.cl/medios/wp-content/uploads/2016/01/Capitulo_8-Scherman_Arriagada_y_Valenzuela_pp_181199-libre.pdf

\section{Entrevistas}

Arancibia, Natalia. Subdirectora de la Agencia Chilena de Eficiencia Energética (AChEE) el 11 noviembre 2014.

Aguirre, Anita. División de Eficiencia Energética. Ministerio de Energía. 11 de noviembre 2014.

Dufey, Annie. Jefe división prospectiva y política energética, Ministerio de Energía. 7 noviembre 2014.

Padilla, Marcelo, División de Eficiencia Energética. Ministerio de Energía. 7 noviembre 2014.

Urquiza, Francisco. Coordinador general de sustentabilidad UC, 4 noviembre 2015.

Zulueta, Javier. Jefe Unidad de Participación y diálogo Ministerio de Energía 7 noviembre 2014.

\section{Encuestas}

CEP. Estudio Nacional de Opinión Pública, Noviembre-Diciembre 2010. Incluye tema especial: Medio Ambiente ISSP 2010, Chile, Centro de Estudios Públicos. Recuperado de: http://www.cepchile.cl/1_4727/doc/ estudio_nacional_de_opinion_publica_noviembre-diciembre_2010_incluye_tema_esp.html 
CEP. Estudio Nacional de Opinión Pública: Diversas Encuestas desde 2000-2013, Santiago de Chile, Centro de Estudios Públicos. Recuperado de: www.cepchile.cl/bannerscep/bdatos_encuestas_cep/base_datos.php

GFK Adimark, Evaluación de Gobierno (2011-2015), Chile, GFK Adimark. Recuperado de:

GFK Adimark, Reciclaje ¿Qué tanto nos interesa? 2015, Chile, GFK Adimark. Recuperado de: http:// www.adimark.cl/es/estudios/index.asp?id=268

IPSOS. Encuestas (2011-2013), Chile, IPSOS. Recuperado de: http://www.ipsos.cl/

IPSOS. Estudio del conocimiento de la hora del planeta 2013, Chile, Ipsos Public Affairs; WWF. Recuperado de: http://d2ouvy59p0dg6k.cloudfront.net/downloads/ipsos_wwf__la_hora_del_planeta.pdf

CADEM. Primera Encuesta Nacional De Medio Ambiente 2014, Chile, CADEM S.A; Ministerio de Medio Ambiente

CADEM. Sexta Encuesta Nacional de Accesos, Usos y Usuarios de Internet en Chile 2015. Chile, CADEM S.S; SUBTEL

UNAB y CEC. Encuesta sobre actitudes hacia el medio ambiente 2011-2014, Chile, UNAB; CEC. Recuperado de:

UNAB y CEC (2012). Comparación de resultados regionales 2011-2012.

UDP Encuesta Nacional (2014 - 2015), Chile, Universidad Diego Portales. Recuperado de: http:// sustentabilidad.unab.cl/encuestas-medio-ambiente/

UDP Encuesta Jóvenes, participación y consumo de medios 2011, Chile, UDP Periodismo; Feedback 